\title{
Inteligência: Efeito do Tipo de Escola e Implicações na Normatização das Escalas Wechsler para Crianças
}

\author{
Santuza Fernandes Silveira Cavalini \\ Centro de Ciências Biológicas e da Saúde da Universidade Presbiteriana Mackenzie, \\ São Paulo, SP, Brasil \\ Tatiana Pontrelli Mecca \\ Laboratório de Neurociência Cognitiva e Social e Clínica de Transtornos do Espectro \\ do Autismo da Universidade Presbiteriana Mackenzie, São Paulo, SP, Brasil \\ Programa de Pós-Graduação em Psicologia Educacional do Centro Universitário \\ FIEO - Unifieo, Osasco, SP, Brasil \\ Cyntia Pinheiro \\ Camila Cruz-Rodrigues \\ Centro de Ciências Biológicas e da Saúde da Universidade Presbiteriana Mackenzie, \\ São Paulo, SP, Brasil \\ Elizeu Coutinho de Macedo ${ }^{1}$ \\ Programa de Pós-Graduação em Distúrbio do Desenvolvimento da Universidade \\ Presbiteriana Mackenzie, São Paulo, SP, Brasil
}

\begin{abstract}
Resumo
A capacidade intelectual dos indivíduos está relacionada a diversos fatores, entre eles o nível socioeconômico. Desta forma, o presente estudo teve por objetivo comparar o desempenho de crianças de escolas públicas e particulares na Escala de Inteligência Wechsler para Crianças - $3^{\mathrm{a}}$ edição (WISC-III). Foram avaliadas 296 crianças entre 6 e 9 anos de idade. Os resultados mostraram desempenho superior dos estudantes de escolas particulares nos subtestes específicos, nos índices fatoriais e gerais, com diferenças estatisticamente significativas entre os grupos etários. Foi verificado um tamanho de efeito maior para os subtestes verbais, uma vez que estes estão associados a experiências formais de aprendizagem. De modo geral, as diferenças se mantiveram ao longo das faixas etárias, exceto para o subteste Completar Figuras e para os subtestes Código e Procurar Símbolos. Nestes últimos as diferenças foram observadas apenas aos 6 e 7 anos de idade. O presente estudo aponta para a importância das diferenças entre tipos de escolas no desempenho em tarefas cognitivas, que devem ser consideradas na normatização de instrumentos como as Escalas Wechsler de Inteligência.
\end{abstract}

Palavras-chave: Inteligência, nível socioeconômico, escola pública, escola particular.

1 Endereço para correspondência: Laboratório de Neurociência Cognitiva e Social, Rua Piauí, 181, 10ªndar, Consolação, São Paulo, SP, Brasil 01302-000. E-mail: santuza.cavalini@mackenzie.br, tati.mecca@gmail. com,chhrpin@gmail.com, camilacruzrodrigues@hotmail.com e elizeumacedo@uol.com.br Apoio financeiro: Fundação de Amparo à Pesquisa do Estado de São Paulo (FAPESP), Conselho Nacional de Desenvolvimento Científico e Tecnológico (CNPq). 


\title{
Intelligence: School Effects and Implications for Standardization of Wechsler Scales for Children
}

\begin{abstract}
The individual's intellectual ability is related to several factors, including socioeconomic status. The presente study aimed to compare the performance of children in public and private schools on the Wechsler Intelligence Scale for Children - $3^{\text {rd }}$ edition (WISC-III) in 296 children between 6 and 9 years old. The results showed superior performance of private school students in specific subtests and general indexes, with statistically significant differences between age groups. A larger effect on verbal subtests was checked, since these are associated with formal learning experiences. Overall differences remained over the age groups, except for the Picture Completion Coding and Search Symbols subtests. These latter differences were observed only at 6 and 7 years old. This study points to the importance of the differences between types of schools on performance in cognitive tasks, which should be considered in the standardization of instruments such as the Wechsler Intelligence Scales
\end{abstract}

Keywords: Intelligence, socioeconomic factor, public schools, private schools.

\section{Inteligencia: Efecto del Tipo de Escuela y Implicaciones sobre la Normalización de las Escalas Wechsler para Niños}

\section{Resumen}

La capacidad intelectual de las personas está relacionada con varios factores, como el nivel socioeconómico. Este estudio tuvo como objetivo comparar el rendimiento de los niños en las escuelas públicas y privadas en el Escala de Inteligencia de Weschler para Niños (WISC-III) en 296 niños de entre 6 y 9 años de edad. Los resultados mostraron un rendimiento superior de los estudiantes de escuelas privadas en subtests específicos en los factores y los índices generales, con diferencias estadísticamente significativas entre los grupos de edad. Un efecto mayor en subtests verbales se comprobó, ya que estos se asocian con experiencias formales de aprendizaje. Las diferencias globales se mantuvieron en los grupos de edad, excepto en los subtest cifras completas y subtests Símbolos y búsqueda de códigos. Se observó que estas últimas diferencias sólo a los 6 y 7 años de edad. Este estudio apunta a la importancia de las diferencias entre los tipos de escuelas en el rendimiento en tareas cognitivas, que deben ser considerados en la estandarización de los instrumentos como las Escalas Wechsler de Inteligencia

Palabras clave: Inteligencia, nivel socioeconômico, escuelas públicas, escuelas privadas.

A capacidade intelectual está relacionada a fatores genéticos e ambientais (Nisbett et al., 2012). Em algumas versões das escalas Wechsler, por exemplo, há diferenças de desempenho considerando as variáveis étnicas (Flanagan \& Kaufman, 2004), ocupacionais e de gênero (Loehlin, 2000).

Uma revisão realizada por Nisbett et al. (2012) analisou os efeitos dos fatores sociais e do ambiente familiar sobre o QI, mostrando que as diferenças de classes socioeconômicas e raciais são fatores preponderantes para o desenvolvimento das habilidades intelectuais e nos processos de socialização das crianças. Sendo assim, quanto maior o nível socioeconômico dos pais, maior o repertório de vocabulário adquirido pelos filhos. Embora considerem o ambiente familiar um fator relevante para o desenvolvimento cognitivo, esses autores ressaltam que os aspectos genéticos e ambientais não são completamente dissociados.

No que tange aos aspectos ambientais, Flores-Mendoza e Nascimento (2007) compararam o desempenho de 59 crianças entre 7 e 8 anos da zona rural, cuja situação socioeconômica se caracterizava como precária, com 143 crianças 
da zona urbana. Os resultados indicaram piores desempenhos no grupo de crianças da zona rural tanto em inteligência fluida, quanto nos subtestes Aritmética e Dígitos da WISC-III que avaliam memória operacional verbal. No entanto, observou-se maior tamanho de efeito da diferença entre os grupos nas Matrizes Progressivas Coloridas de Raven - Escala Especial, indicando que um ambiente empobrecido afetou com maior intensidade a inteligência fluida em relação à cristalizada. A inteligência fluida se refere à capacidade de raciocínio em situações novas, pensar de maneira abstrata, relacionar conceitos de modo a resolver problemas eficientemente, entretanto a inteligência cristalizada se refere à capacidade de raciocínio a partir de conhecimentos previamente adquiridos a partir de situações de aprendizagem (Carroll, 2012). Atualmente esta dimensão da inteligência é denominada de "compreensão/conhecimento" e abrange habilidades específicas distintas como informações verbais gerais, desenvolvimento da linguagem, conhecimento lexical, compreensão de um discurso, entre outras que são adquiridas, consolidadas e influenciadas pela cultura e educação (Schneider \& McGrew, 2012).

No entanto, esse quadro pode ser revertido a partir de procedimentos que tornam o ambiente mais enriquecido, possibilitando o desenvolvimento da capacidade de raciocínio. Klauer e Phye (1995) mostram que é possível estimular a inteligência fluida, por exemplo, através de um programa de atividades de raciocínio indutivo e solução de problemas. De acordo com estes autores, habilidades como generalização, discriminação e o reconhecimento de semelhanças e diferenças entre estímulos influenciam no potencial para a aprendizagem e no desenvolvimento de habilidades para solução de problemas.

O ensino formal também pode ser uma possibilidade de estimulação, já que o tempo de escolarização é uma das variáveis que se relaciona com o desenvolvimento cognitivo (Mecca, 2010). Esta autora comparou crianças com a mesma faixa etária e pertencentes ao mesmo estágio do Ensino Infantil de escolas públicas, mas com diferenças de seis meses no tempo de escolarização. Os resultados mostraram diferen- ças significativas de desempenho em tarefas de raciocínio indutivo, discriminação, exploração e síntese visual com maiores escores no grupo com 6 meses a mais de escolarização. No caso das crianças e adolescentes, os anos de escolaridade são fortemente correlacionados com a idade e impactam diretamente na capacidade intelectual (Primi, Couto, Almeida, Guisande, \& Miguel, 2012).

Sabe-se que o tipo de escola, pública ou particular é uma variável que também possui influência nos escores de testes de inteligência (Neisser, 1998). A partir dos resultados de estudos nacionais é possível observar diferenças no desempenho entre crianças de escolas públicas e de escolas particulares em diferentes instrumentos que avaliam habilidades específicas da inteligência em idades distintas (Almeida \& Primi, 2000; Alves \& Duarte, 2001; Angelini, Alves, Custódio, Duarte, \& Duarte, 1999; Bandeira, Alves, Giacomel, \& Lorenzatto, 2004). Sendo assim, é fundamental compreender o efeito do tipo de escola no desenvolvimento cognitivo das crianças brasileiras, já que no Brasil, de acordo com a Pesquisa Nacional de Saúde do Escolar - PeNSE 2012 (Instituto Brasileiro de Geografia e Estatística [IBGE], 2012) 82,8\% da população em idade escolar frequenta a escola pública.

Uma das diferenças importantes entre crianças de escolas públicas e particulares verificadas pelo IBGE é o nível de escolaridade das mães. Dados recentes indicam que há maior prevalência de mães de crianças pertencentes a escolas públicas que não possuem instrução ou que não finalizaram o Ensino Fundamental. Trata-se de um indicador importante do nível socioeconômico (IBGE, 2012). Estudos mostram que há uma relação entre nível de escolaridade dos pais e habilidades cognitivas dos filhos (Carneiro, Meghir, \& Parey, 2013; Vista \& Grantham, 2010).

Vista e Grantham (2010) investigaram a influência do nível educacional dos pais na inteligência fluida dos filhos. Estes autores observaram uma progressão de cerca de 3 pontos no QI dos filhos de acordo com o aumento nos níveis de escolaridade dos pais em relação ao Ensino Médio, Graduação e Pós-graduação. No entanto, maior tamanho de efeito é observado ao compa- 
rar o nível intelectual de crianças cujo nível educacional dos pais é graduação ou pós-graduação com aquelas cujos pais não terminaram o ensino fundamental. Além desses, o estudo de Carneiro et al. (2013) investigou a influência do nível educacional das mães no desempenho acadêmico dos filhos e constatou que a diferença de um ano a mais de escolaridade materna está associado a uma diferença de 5 pontos percentuais nos resultados de testes dos seus filhos.

Os dados do IBGE (2012) mostram que estudantes de escolas públicas e particulares apresentam condições socioeconômicas diferentes. Em testes de inteligência também se observam desempenhos diferentes, com maiores médias em crianças de escolas particulares. Estas diferenças influenciam diretamente na elaboração de normas específicas para cada tipo de escola, que constam nos manuais técnicos de alguns instrumentos brasileiros como as Matrizes Progressivas Coloridas de Raven - Escala Especial (Angelini et al., 1999; Bandeira et al., 2004), a Bateria de Provas de Raciocínio (Almeida \& Primi, 2000) e a Escala de Maturidade Mental Colúmbia (Alves \& Duarte, 2001). A elaboração de normas distintas para crianças de escolas públicas e particulares são necessárias, pois proporcionam a interpretação adequada dos resultados obtidos, considerando o contexto escolar e social no qual a criança está inserida.

Entretanto, apenas alguns instrumentos que avaliam a capacidade intelectual apresentam normas distintas em função do tipo de esco- la. No caso das versões brasileiras das Escalas Wechsler de Inteligência, como a WISC-III (Figueiredo, 2002) e mais recentemente a WISC-IV (Rueda, Noronha, Sisto, Santos, \& Castro, 2013) não há dados normativos distintos para escolas públicas e particulares, embora seja uma das escalas mais utilizadas para a avaliação de inteligência em crianças e adolescentes (Moura, Simões, \& Pereira, 2014). Neste sentido, é necessária a condução de estudos que objetivam verificar e compreender diferenças de desempenho na WISC entre alunos de escolas públicas e particulares. Considerando o que foi exposto, o objetivo do presente estudo foi comparar o desempenho na WISC-III entre crianças de escolas públicas e particulares, pertencentes ao Ensino Fundamental I.

\section{Método}

\section{Participantes}

Participaram do estudo 296 crianças entre 6 e 9 anos de idade $(M=7,60 ; D P=1,10)$. A caracterização da amostra é apresentada na Tabela 1 em função das variáveis: idade, sexo e tipo de escola (pública ou particular). Foram incluídos sujeitos sem queixas de dificuldades de aprendizagem, atraso no desenvolvimento neuropsicomotor ou problemas de comportamento de acordo com uma entrevista de anamnese feita com os responsáveis para levantamento de informações referentes ao desenvolvimento neuropsicomotor e acadêmico da criança.

Tabela 1

Descrição da Amostra de Acordo com Idade e Sexo em Função do Tipo de Escola

\begin{tabular}{ccc}
\hline & Pública (\%) & Particular (\%) \\
\hline Idades & $123(41,6)$ & $173(58,4)$ \\
6 & & $37(21,4)$ \\
7 & $24(19,5)$ & $39(22,5)$ \\
8 & $39(31,7)$ & $47(27,2)$ \\
9 & $27(22,0)$ & $50(28,9)$ \\
Sexo & $33(26,8)$ & \\
Feminino & & $89(51,4)$ \\
Masculino & $62(54,4)$ & $84(48,6)$ \\
\hline
\end{tabular}




\section{Instrumento}

Utilizou-se a $3^{\text {a }}$ edição das Escalas Wechsler de Inteligência para Crianças (WISC-III) (Figueiredo, 2002). Trata-se de um instrumento que avalia o funcionamento intelectual geral a partir de dez subtestes obrigatórios e três subtestes complementares agrupados em índices fatoriais conforme apresentado na Tabela 2. As normas para população brasileira estão disponíveis em função das faixas etárias entre crianças de 6 a 16 anos de idade. Foram aplicados todos os subtestes, com exceção de Labirintos.

Tabela 2

Subtestes e Índices Fatoriais da WISC-III

\begin{tabular}{ccccccc}
\hline & & & & & QI Total \\
\hline Subtestes & CV & RD & OP & VP & QI Verbal & QI Execução \\
Informação & $\sqrt{ }$ & & & $\sqrt{ }$ & \\
Semelhanças & $\sqrt{ }$ & & & $\sqrt{ }$ & \\
Vocabulário & $\sqrt{ }$ & & & $\sqrt{ }$ & \\
Compreensão & $\sqrt{ }$ & & & $\sqrt{ }$ & \\
Aritmética & & $\sqrt{ }$ & & & $\sqrt{ }$ \\
Dígitos & & $\sqrt{ }$ & & & $\sqrt{ }$ \\
Completar Figuras & & & $\sqrt{ }$ & $\sqrt{ }$ \\
Arranjo de Figuras & & & $\sqrt{ }$ & & \\
Cubos & & & & & $\sqrt{ }$ \\
Armar Objetos & & & & & \\
Código & & & & & \\
Procurar Símbolos & & & & & \\
\hline
\end{tabular}

Nota. Legenda: Compreensão Verbal - CV; Resistência à Distração - RD; Organização Perceptual - OP; Velocidade de Processamento - VP.

\section{Procedimento}

Para a coleta de dados foram convidados alunos de escolas públicas e particulares de diferentes regiões da cidade de São Paulo. Os responsáveis pelas crianças, que aceitaram participar da pesquisa, assinaram o termo de consentimento livre e esclarecido, contemplando os procedimentos éticos necessários para sua participação no estudo. As avaliações ocorreram individualmente em salas de aplicação de testes do Serviço Escola de uma universidade em São Paulo, durante dois encontros de 50 minutos cada. No primeiro aplicaram-se os seis primeiros subtestes da WISC III e no segundo, os seis subtestes restantes.

Para análise dos resultados utilizou-se o programa Statistical Package for Social Sciences ${ }^{\circledR}$ versão 18.0 para Windows (SPSS Inc.). O nível de significância adotado foi de $5 \%$ para todas as análises. Realizou-se análise de variância univariada (ANOVA) para comparação do desempenho entre crianças de escolas públicas e particulares para as diferentes faixas etárias e para a amostra total. Foi verificado o tamanho de efeito das diferenças observadas a partir da fórmula de $d$ de Cohen. De acordo com este autor considera-se: $0,29=$ pequeno; 0,30 a $0,79=$ médio $; \geq 0,80$ $=$ grande. Consideraram-se as pontuações ponderadas para as análises envolvendo os subtestes específicos e os escores padronizados em QI para as análises dos índices globais (QI Verbal, QI Execução e QI Total) e fatoriais (Compreensão Verbal, Organização Perceptual, Resistência à Distração e Velocidade de Processamento). 


\section{Resultados}

A fim de verificar a existência de diferenças nas idades entre alunos pertencentes a escolas públicas e particulares, foi realizado Teste $t$ de Student para amostras independentes. Os resultados revelaram que estudantes de escolas públicas $(M=7,56 ; D P=1,08)$ e estudantes de escolas particulares $(M=7,64 ; D P=1,11)$ apresentaram médias semelhantes em relação à idade, sendo que não há diferença estatisticamente significativa entre os grupos, $t(294)=0,075 ; p$ $=0,566$.

Em seguida procedeu-se à comparação do desempenho entre os tipos de escola, de acordo com as diferentes faixas etárias nos índices fatoriais e globais da WISC-III. Os resultados obtidos a partir da análise de variância e o tamanho de efeito encontram-se sumariados na Tabela 3.

As estatísticas descritivas revelam médias superiores no grupo de crianças pertencentes a escolas particulares em todas as medidas globais e índices fatoriais da WISC-III. Ao considerar as interpretações de acordo com o QI, pode-se observar que no QI Verbal, as crianças de 6 a 8 anos de escolas públicas apresentaram desempenho médio e o grupo com 9 anos apresentou desempenho médio superior. Enquanto que as crianças de escolas particulares apresentaram desempenho médio superior aos 6 anos e superior dos 7 aos 9 anos de idade. Houve diferenças estatisticamente significativas no QI Verbal entre os tipos de escolas em todas as faixas etárias e na amostra total. Essas diferenças se mantém estáveis ao longo das faixas etárias, ou seja, não há aumento ou diminuição conforme o desenvolvimento e a escolarização. Os valores obtidos a partir da fórmula de $\mathrm{d}$ de Cohen indicaram tamanho de efeito grande para as diferenças observadas.

Em relação ao QI de Execução, crianças dos 6 aos 8 anos de escolas públicas apresentaram QI médio, enquanto que aos 9 anos, o QI foi médio superior, assim como observado no QI Verbal. Já o grupo de escolas particulares, apresentou QI de Execução médio superior dos 6 aos 8 anos e superior aos 9 anos de idade. Também foram verificadas diferenças estatisticamente significativas entre todos os grupos etários e a amostra total, com tamanho de efeito grande aos 6 anos e médio nas demais faixas etárias. De acordo com as médias obtidas em cada faixa etária, as diferenças entre os tipos de escola são maiores aos 6 anos e diminuem nas demais idades.

O mesmo padrão observado no QI de Execução, também foi verificado ao longo das idades no QI Total, ou seja, crianças dos 6 aos 8 anos de escolas públicas apresentaram QI médio e médio superior aos 9 anos, enquanto que o grupo de escolas particulares apresentou QI de Execução médio superior dos 6 aos 8 anos e superior aos 9 anos de idade. As diferenças entre os grupos foram estatisticamente significativas e permaneceram semelhantes e estáveis de acordo com o aumento das idades e consequentemente do nível de escolarização. Tamanho de efeito grande foi verificado para as diferenças entre os tipos de escola em todas as idades.

Ao investigar os índices fatoriais, observou-se que em Compreensão Verbal crianças dos 6 aos 8 anos de escolas públicas apresentam desempenho médio e médio superior aos 9 anos. No grupo de crianças de escolas particulares verificou-se desempenho médio superior aos $6 \mathrm{e}$ 7 anos, enquanto que aos 8 e 9 anos, o desempenho foi superior. As diferenças entre os grupos foram estatisticamente significativas e se mantiveram estáveis conforme o aumento das idades, com grande tamanho de efeito.

No índice de Organização Perceptual crianças dos 6 aos 8 anos de escolas públicas apresentaram desempenho médio e aos 9 anos, médio superior. Já nas escolas particulares foram verificados dos 6 aos 8 anos desempenho médio superior e aos 9 anos de idade, superior. Foram observadas diferenças estatisticamente significativas aos 6, 8 e 9 anos de idade, bem como uma tendências aos 7 anos $(p=0,070)$ entre alunos escolas públicas e particulares, com grande tamanho de efeito aos 6 anos e moderado nas demais faixas etárias. Além disso, em ambos os grupos há uma estabilidade em relação à habilidade de organização perceptual dos 6 aos 8 anos em ambos os tipos de escola, além de um aumento dos 
Tabela 3

Comparação do Desempenho entre Alunos de Escolas Públicas e Particulares

\begin{tabular}{|c|c|c|c|c|c|}
\hline Variáveis & Idade & Escola Pública & Escola Particular & $F$ & $d$ \\
\hline \multirow[t]{5}{*}{ QI Verbal } & 6 & $98,00( \pm 10,29)$ & $114,89( \pm 13,87)$ & $24,451 * *$ & 1,34 \\
\hline & 7 & $103,13( \pm 16,78)$ & $120,49( \pm 12,39)$ & $27,003 * *$ & 1,18 \\
\hline & 8 & $107,19( \pm 18,57)$ & $121,45( \pm 13,14)$ & $14,848 * *$ & 0,93 \\
\hline & 9 & $113,75( \pm 17,56)$ & $128,54( \pm 13,04)$ & $19,065^{* *}$ & 0,99 \\
\hline & Total & $105,80( \pm 17,12)$ & $121,74( \pm 13,93)$ & $81,415^{*}$ & 1,04 \\
\hline \multirow[t]{5}{*}{ QI Execução } & 6 & $103,13( \pm 12,44)$ & $118,41( \pm 15,24)$ & $16,805^{* *}$ & 1,08 \\
\hline & 7 & $108,05( \pm 13,85)$ & $116,13( \pm 12,40)$ & $7,356 * *$ & 0,61 \\
\hline & 8 & $102,74( \pm 12,51)$ & $112,37( \pm 14,39)$ & $8,364 * *$ & 0,70 \\
\hline & 9 & $111,79( \pm 12,36)$ & $120,82( \pm 12,78)$ & $10,178 * *$ & 0,72 \\
\hline & Total & $106,98( \pm 13,30)$ & $116,74( \pm 13,87)$ & $35,724 *$ & 0,72 \\
\hline \multirow[t]{5}{*}{ QI Total } & 6 & $100,13( \pm 11,03)$ & $117,36( \pm 13,17)$ & $27,964 * *$ & 1,39 \\
\hline & 7 & $105,90( \pm 14,61)$ & $119,49( \pm 11,84)$ & $20,353 * *$ & 1,02 \\
\hline & 8 & $105,44( \pm 14,64)$ & $118,34( \pm 13,18)$ & $15,130 * *$ & 0,94 \\
\hline & 9 & $114,13( \pm 14,44)$ & $126,98( \pm 12,43)$ & $18,375^{* *}$ & 0,97 \\
\hline & Total & $106,82( \pm 14,61)$ & $120,85( \pm 13,09)$ & $76,116^{*}$ & 1,02 \\
\hline \multirow[t]{5}{*}{ Compreensão Verbal } & 6 & $96,75( \pm 11,21)$ & $113,54( \pm 15,75)$ & $20,476^{* *}$ & 1,19 \\
\hline & 7 & $102,00( \pm 17,07)$ & $119,46( \pm 15,62)$ & $22,203 * *$ & 1,07 \\
\hline & 8 & $105,30( \pm 18,85)$ & $122,49( \pm 15,38)$ & $18,125^{* *}$ & 1,03 \\
\hline & 9 & $114,16( \pm 20,15)$ & $129,10( \pm 13,41)$ & $16,093 * *$ & 0,91 \\
\hline & Total & $104,89( \pm 18,28)$ & $121,71( \pm 15,92)$ & $14,928 *$ & 0,99 \\
\hline \multirow[t]{5}{*}{ Organização Perceptual } & 6 & $101,42( \pm 13,34)$ & $116,27( \pm 15,88)$ & $14,376^{* *}$ & 0,99 \\
\hline & 7 & $106,90( \pm 14,26)$ & $112,90( \pm 14,54)$ & 3,383 & 0,42 \\
\hline & 8 & $102,63( \pm 13,20)$ & $113,02( \pm 15,27)$ & $8,738 * *$ & 0,71 \\
\hline & 9 & $110,52( \pm 14,68)$ & $120,90( \pm 12,79)$ & $11,639 * *$ & 0,76 \\
\hline & Total & $105,88( \pm 14,31)$ & $115,82( \pm 14,55)$ & $33,088^{*}$ & 0,69 \\
\hline \multirow[t]{5}{*}{ Resistência à Distração } & 6 & $100,42( \pm 14,52)$ & $113,58( \pm 10,58)$ & $16,509 * *$ & 1,07 \\
\hline & 7 & $101,95( \pm 13,34)$ & $115,08( \pm 14,70)$ & $17,052 * *$ & 0,94 \\
\hline & 8 & $106,52( \pm 15,94)$ & $112,94( \pm 12,97)$ & 3,541 & 0,45 \\
\hline & 9 & $108,91( \pm 14,78)$ & $119,60( \pm 11,92)$ & $13,181 * *$ & 0,81 \\
\hline & Total & $104,48( \pm 14,82)$ & $115,36( \pm 12,79)$ & $44,622^{*}$ & 0,43 \\
\hline \multirow{5}{*}{$\begin{array}{l}\text { Velocidade de } \\
\text { Processamento }\end{array}$} & 6 & $103,83( \pm 12,84)$ & $117,68( \pm 16,58)$ & $12,020 * *$ & 0,91 \\
\hline & 7 & $103,95( \pm 18,96)$ & $115,69( \pm 13,75)$ & $9,800 * *$ & 0,71 \\
\hline & 8 & $93,85( \pm 20,64)$ & $104,77( \pm 17,54)$ & $5,828 *$ & 0,58 \\
\hline & 9 & $106,79( \pm 14,43)$ & $110,56( \pm 17,02)$ & 1,097 & 0,24 \\
\hline & Total & $102,60( \pm 17,65)$ & $111,48( \pm 17,09)$ & $19,489 *$ & 0,85 \\
\hline
\end{tabular}


8 para os 9 anos de idade também em ambos os grupos.

No índice fatorial de Resistência à Distração houve diferenças estatisticamente significativas entre os grupos aos 6, 7 e 9 anos de idade, com grande tamanho de efeito. Já aos 8 anos foi observada uma tendência $(p=0,064)$, com tamanho de efeito moderado. Em todas as faixas etárias as crianças pertencentes às escolas públicas apresentaram desempenho na média enquanto que nas escolas particulares foi médio superior. Ambas foram semelhantes em relação à estabilidade da capacidade de resistência à distração ao longo das faixas etárias.

Por fim, no índice de Velocidade de Processamento, crianças de escolas públicas apresenta- ram desempenho médio assim como crianças de 8 anos de escolas particulares. Nestas, crianças de outras faixas etárias apresentaram desempenho médio superior. Diferenças estatisticamente significativas foram verificadas entre os tipos de escola nos grupos com 6 a 8 anos de idade, exceto aos 9 anos $(p=0,298)$. Tamanho de efeito grande foi observado entre os grupos com 6 anos, e moderado entre os grupos com 7 e 8 anos.

Em seguida foram comparados os desempenhos entre os tipos de escola de acordo com cada subteste. Primeiramente foram analisados os subtestes não-verbais, ou seja, de execução. As estatísticas descritivas, os resultados obtidos a partir da ANOVA e a magnitude do efeito encontram-se descritos na Tabela 4.

\section{Tabela 4}

Comparação entre Escolas Públicas e Particulares nos Subtestes Não-Verbais da WISC-III

\begin{tabular}{|c|c|c|c|c|c|}
\hline Subtestes & Idade & Escola Pública & Escola Particular & $F$ & $d$ \\
\hline \multirow[t]{5}{*}{ Completar Figuras } & 6 & $12,25( \pm 2,93)$ & $13,43( \pm 2,55)$ & 2,77 & 0,44 \\
\hline & 7 & $13,26( \pm 2,85)$ & $13,97( \pm 2,52)$ & 1,383 & 0,26 \\
\hline & 8 & $11,04( \pm 1,78)$ & $12,87( \pm 2,47)$ & $11,325 * *$ & 0,82 \\
\hline & 9 & $12,88( \pm 2,58)$ & $13,58( \pm 2,83)$ & 1,303 & 0,26 \\
\hline & Total & $12,47( \pm 2,70)$ & $13,45( \pm 2,62)$ & $9,695 * *$ & 0,37 \\
\hline \multirow[t]{5}{*}{ Código } & 6 & $10,71( \pm 2,97)$ & $13,24( \pm 3,26)$ & $9,422 * *$ & 0,8 \\
\hline & 7 & $10,90( \pm 3,53)$ & $13,00( \pm 3,22)$ & $7,534 * *$ & 0,62 \\
\hline & 8 & $8,70( \pm 3,22)$ & $10,34( \pm 3,74)$ & 3,610 & 0,46 \\
\hline & 9 & $11,00( \pm 3,02)$ & $11,42( \pm 2,80)$ & 0,419 & 0,15 \\
\hline & Total & $10,41( \pm 3,31)$ & $11,87( \pm 3,45)$ & $13,402 * *$ & 0,43 \\
\hline \multirow[t]{5}{*}{ Arranjo de Figuras } & 6 & $9,58( \pm 2,30)$ & $11,86( \pm 3,07)$ & $9,674 * *$ & 0,82 \\
\hline & 7 & $10,13( \pm 2,92)$ & $11,64( \pm 2,74)$ & $5,548 *$ & 0,53 \\
\hline & 8 & $11,30( \pm 3,22)$ & $12,36( \pm 3,56)$ & 1,644 & 0,31 \\
\hline & 9 & $11,97( \pm 2,86)$ & $13,42( \pm 3,25)$ & $4,325^{*}$ & 0,47 \\
\hline & Total & $10,77( \pm 2,98)$ & $12,40( \pm 3,24)$ & $19,286^{* *}$ & 0,52 \\
\hline \multirow[t]{5}{*}{ Cubos } & 6 & $10,92( \pm 3,40)$ & $13,95( \pm 3,97)$ & $9,453 * *$ & 0,81 \\
\hline & 7 & $11,36( \pm 3,33)$ & $12,90( \pm 3,03)$ & $4,540 *$ & 0,48 \\
\hline & 8 & $10,52( \pm 3,17)$ & $12,02( \pm 3,37)$ & 3,54 & 0,45 \\
\hline & 9 & $12,03( \pm 3,42)$ & $14,28( \pm 2,77)$ & $10,652 * *$ & 0,74 \\
\hline & Total & $11,26( \pm 3,34)$ & $13,28( \pm 3,38)$ & $25,798 * *$ & 0,6 \\
\hline
\end{tabular}




$\begin{array}{lccccc}\text { Armar Objetos } & 6 & 9,00( \pm 2,41) & 10,76( \pm 2,67) & 6,598^{*} & 0,69 \\ & 7 & 9,90( \pm 2,78) & 10,03( \pm 2,99) & 0,038 & 0,05 \\ \text { Procurar Símbolos } & 8 & 9,70( \pm 2,70) & 11,15( \pm 2,66) & 5,002^{*} & 0,54 \\ & 9 & 10,70( \pm 2,59) & 11,90( \pm 2,32) & 4,861^{*} & 0,39 \\ & \text { Total } & 9,90( \pm 2,68) & 11,03( \pm 2,71) & 34,876^{* *} & 0,42 \\ & 6 & 11,17( \pm 3,00) & 13,62( \pm 3,36) & 8,403^{* *} & 0,76 \\ & 7 & 11,51( \pm 2,57) & 13,49( \pm 2,07) & 13,904^{* *} & 0,85 \\ & 9 & 10,56( \pm 2,57) & 11,79( \pm 3,32) & 2,752 & 0,4 \\ & \text { Total } & 11,79( \pm 3,33) & 13,16( \pm 3,10) & 3,66 & 0,43 \\ & 11,31( \pm 2,88) & 12,96( \pm 3,09) & 21,656^{* *} & 0,55\end{array}$

${ }^{*} p \leq 0,05 ; * * p \leq 0,01$.

De acordo com os dados apresentados na Tabela 3, diferenças estatisticamente significativas entre alunos de escolas públicas e particulares foram verificadas somente em determinadas faixas etárias nos subtestes de execução. No subteste Completar Figuras, por exemplo, diferenças significativas foram verificadas somente entre os grupos com 8 anos de idade. Já nos subtestes que requerem velocidade de processamento, Código e Procurar Símbolos, as diferenças foram observadas somente nas faixas etárias iniciais, dos 6 e 7 anos. Nos subtestes Arranjo de Figuras e Cubos, além dos grupos com 6 e 7 anos, diferenças significativas também ocorreram aos 9 anos. Por fim, em Armar Objetos, houve diferenças significativas entre os grupos etários, exceto aos 7 anos de idade. Estes dados mostram que aos 6 anos as diferenças entre escolas públicas e particulares aparecem em 5 dos 6 subtestes (Código, Arranjo de Figuras, Cubos, Armar Objetos e Procurar Símbolos) diminuindo com o aumento das faixas etárias. Aos 7 anos de idade as diferenças entre os grupos são observadas em 4 dos 6 subtestes (Código, Arranjo de Figuras, Cubos e Procurar Símbolos). Já aos 8 e 9 anos, apenas dois subtestes apresentam diferenças significativas em cada faixa etária, sendo Completar Figuras e Armar Objetos aos 8 anos e este último juntamente com Cubos aos 9 anos de idade.

Vale ressaltar que de acordo com o desvio-padrão indicado para análise dos pontos pon- derados dos subtestes $(M=10 ; D P=3,00)$, ambos os grupos apresentaram médias esperadas em relação a sua faixa etária. Isto indica que embora maiores médias tenham sido observadas nos grupos de escolas particulares em relação aos grupos de escolas públicas, estes apresentaram desempenho dentro do esperado de acordo com as normas do instrumento. A seguir, a Tabela 5 apresenta os resultados obtidos a partir das comparações entre escolas públicas e particulares nos subtestes verbais.

Os dados apresentados na Tabela 4 mostram diferenças significativas entre o desempenho de alunos de escolas públicas e particulares em todas as faixas etárias nos subtestes que compõem o índice de Compreensão Verbal: Informação, Semelhanças, Vocabulário e Compreensão. Enquanto que nos subtestes que formam o índice de Resistência à Distração (Aritmética e Dígitos), diferenças significativas foram verificadas na maioria dos grupos etários, exceto aos 8 anos de idade. As magnitudes do efeito das diferenças observadas variaram de moderada a alta.

Além disso, ao considerar a média dos pontos ponderados $(M=10 ; D P=3)$, as crianças de escolas particulares apresentaram desempenho acima da média, em sua maioria aos 8 e 9 anos nos subtestes Semelhanças, Vocabulário, Compreensão e Dígitos. De modo geral, nos subtestes verbais, as diferenças de desempenho entre crianças de escolas públicas e particulares tende a manter-se ao longo das faixas etárias. 
Tabela 5

Comparação entre Escolas Públicas e Particulares nos Subtestes Verbais da WISC-III

\begin{tabular}{|c|c|c|c|c|c|}
\hline Subtestes & Idade & Escola Pública & Escola Particular & $F$ & $d$ \\
\hline \multirow[t]{5}{*}{ Informação } & 6 & $8,79( \pm 2,34)$ & $11,89( \pm 3,53)$ & $14,344 * *$ & 0,99 \\
\hline & 7 & $9,56( \pm 2,38)$ & $12,18( \pm 2,97)$ & $18,387 * *$ & 0,97 \\
\hline & 8 & $9,58( \pm 3,56)$ & $11,80( \pm 2,57)$ & $11,325 * *$ & 0,75 \\
\hline & 9 & $11,03( \pm 3,27)$ & $13,90( \pm 3,03)$ & $16,712 * *$ & 0,92 \\
\hline & Total & $9,81( \pm 2,98)$ & $12,52( \pm 3,12)$ & $55,420 * *$ & 0,88 \\
\hline \multirow[t]{5}{*}{ Semelhanças } & 6 & $9,42( \pm 3,45)$ & $13,30( \pm 3,44)$ & $18,436^{* *}$ & 1,13 \\
\hline & 7 & $9,85( \pm 3,88)$ & $13,00( \pm 4,14)$ & $12,013 * *$ & 0,79 \\
\hline & 8 & $11,22( \pm 4,25)$ & $15,11( \pm 3,25)$ & $19,421 * *$ & 1,07 \\
\hline & 9 & $12,70( \pm 4,25)$ & $15,80( \pm 3,16)$ & $14,477 * *$ & 0,85 \\
\hline & Total & $10,83( \pm 4,15)$ & $14,45( \pm 3,66)$ & $62,714 * *$ & 0,94 \\
\hline \multirow[t]{5}{*}{ Aritmética } & 6 & $9,92( \pm 2,55)$ & $12,68( \pm 2,18)$ & $20,308 * *$ & 1,18 \\
\hline & 7 & $10,41( \pm 2,65)$ & $13,03( \pm 2,19)$ & $22,507 * *$ & 1,08 \\
\hline & 8 & $10,78( \pm 2,30)$ & $11,66( \pm 2,20)$ & 2,658 & 0,39 \\
\hline & 9 & $11,27( \pm 2,28)$ & $12,74( \pm 2,18)$ & $8,663 * *$ & 0,66 \\
\hline & Total & $10,63( \pm 2,48)$ & $12,50( \pm 2,23)$ & $45,958 * *$ & 0,80 \\
\hline \multirow[t]{5}{*}{ Vocabulário } & 6 & $10,04( \pm 3,60)$ & $11,95( \pm 3,40)$ & $4,345^{*}$ & 0,55 \\
\hline & 7 & $11,15( \pm 4,67)$ & $14,62( \pm 3,06)$ & $14,969 * *$ & 0,88 \\
\hline & 8 & $11,44( \pm 4,05)$ & $14,57( \pm 3,42)$ & $12,520 * *$ & 0,85 \\
\hline & 9 & $13,12( \pm 4,15)$ & $15,12( \pm 3,02)$ & $6,415^{*}$ & 0,57 \\
\hline & Total & $11,53( \pm 4,29)$ & $14,18( \pm 3,41)$ & $34,876^{* *}$ & 0,70 \\
\hline \multirow[t]{5}{*}{ Compreensão } & 6 & $10,08( \pm 2,94)$ & $12,30( \pm 3,22)$ & $7,340 * *$ & 0,71 \\
\hline & 7 & $11,23( \pm 3,71)$ & $13,54( \pm 2,99)$ & $9,128 * *$ & 0,69 \\
\hline & 8 & $12,07( \pm 3,14)$ & $14,06( \pm 3,98)$ & $4,954^{*}$ & 0,54 \\
\hline & 9 & $12,76( \pm 4,13)$ & $14,80( \pm 2,92)$ & $6,953 * *$ & 0,59 \\
\hline & Total & $11,60( \pm 3,66)$ & $13,78( \pm 3,41)$ & $27,551 * *$ & 0,62 \\
\hline \multirow[t]{5}{*}{ Dígitos } & 6 & $10,71( \pm 3,02)$ & $12,68( \pm 2,17)$ & $8,724 * *$ & 0,78 \\
\hline & 7 & $10,72( \pm 3,12)$ & $13,00( \pm 2,79)$ & $11,556^{* *}$ & 0,77 \\
\hline & 8 & $12,26( \pm 4,30)$ & $13,28( \pm 3,51)$ & 1,216 & 0,27 \\
\hline & 9 & $12,45( \pm 3,55)$ & $14,36( \pm 3,25)$ & $6,343^{*}$ & 0,57 \\
\hline & Total & $11,52( \pm 3,56)$ & $13,40( \pm 3,07)$ & $23,467 * *$ & 0,57 \\
\hline
\end{tabular}

${ }^{*} p \leq 0,05 ; * * p \leq 0,01$

\section{Discussão}

O objetivo do presente estudo foi comparar o desempenho na WISC-III entre crianças de escolas públicas e particulares do Ensino Fundamental I. Ao compreender a inteligência como multidimensional, ou seja, composta por diferentes habilidades (McGrew, 2009), que são 
influenciadas por diferentes fatores, tais como idade e nível socioeconômico e tipo de escola (Neisser, 1998; Nisbett et al., 2012), comparou-se o desempenho de crianças de escolas públicas e particulares de acordo com cada faixa etária nos índices globais, fatoriais e nos subtestes específicos da WISC-III.

Os resultados evidenciaram diferenças significativas no desempenho de crianças oriundas de escolas públicas daquelas matriculadas em escolas particulares, com melhores desempenhos neste último grupo, considerando a amostra total. Estes dados corroboram achados observados em estudos anteriores que utilizaram instrumentos para avaliação da capacidade intelectual em pré-escolares, em crianças do Ensino Fundamental I e adolescentes do Ensino Fundamental II (Almeida \& Primi, 2000; Alves \& Duarte, 2001; Angelini et al., 1999; Bandeira et al., 2004).

Os resultados observados no presente estudo, assim como em outros que utilizaram as Matrizes Progressivas Coloridas de Raven, a Escala de Maturidade Mental Colúmbia e a Bateria de Provas de Raciocínio - BPR5 (Almeida \& Primi, 2000; Alves \& Duarte, 2001; Angelini et al., 1999; Bandeira et al., 2004) indicam que as diferenças de desempenho entre alunos de escolas públicas e particulares, em diferentes testes que demandam habilidades cognitivas distintas, tendem a manter-se independentemente da faixa etária e do instrumento utilizado. Entretanto, vale ressaltar que os estudos anteriores utilizaram instrumentos que avaliam mais especificamente o raciocínio fluido, enquanto que a WISC-III possui muitos subtestes que avaliam a inteligência cristalizada, sendo esta mais relacionada às experiências formais de aprendizagem. Em relação aos subtestes verbais tais como Informação e Vocabulário, Simões (2002) relata a suscetibilidade para o nível de conhecimentos adquiridos na escola, sendo este um fator importante no desempenho de crianças e adolescentes.

De fato, ao considerar as diferenças de desempenho entre os grupos em tarefas verbais e de execução, a magnitude do efeito foi maior no QI Verbal e nos índices de Compreensão Verbal e Resistência à Distração do que as magni- tudes das diferenças observadas no QI de Execução e nos índices de Organização Perceptual e Velocidade de Processamento. Além disso, os dados obtidos pelo $d$ de Cohen mostraram tanto no índice de Organização Perceptual quanto em Velocidade de Processamento, a magnitude do efeito foi maior aos 6 anos de idade, diminuindo conforme o aumento das faixas etárias.

Além de verificar o desempenho geral na WISC-III é fundamental uma análise minuciosa de cada subteste, pois esta permite evidenciar áreas de força ou de déficits específicos (Simões, 2002). Desta forma, procedeu-se a comparação entre os grupos em cada subteste. Ao considerar a amostra total, o desempenho em todos os subtestes da WISC-III revelou-se superior no grupo de crianças pertencentes a escolas particulares em relação ao grupo de crianças das escolas públicas.

As diferenças mostraram um tamanho de efeito médio ( $d$ de Cohen) para todos os subtestes da WISC-III, com exceção do subteste Semelhanças, no qual foi observado um tamanho de efeito alto. Este subteste avalia a habilidade de estabelecer relações lógicas e formar categorias, ou seja, compreender conceitos e integrar conhecimentos (Simões, 2002) tanto de ordem concreta quanto abstrata (Mader, Thais, \& Ferreira, 2004). De acordo com estas autoras o desempenho nesta tarefa está fortemente relacionado às oportunidades culturais disponíveis para $o$ indivíduo.

Diferenças estatisticamente significativas entre os grupos foram observadas na maioria das faixas etárias para cada subteste de execução, sendo que crianças de diferentes faixas etárias pertencentes a escolas particulares apresentam desempenho superior no que tange as habilidades relacionadas a capacidades perceptivas como organização, planejamento e sequenciamento de histórias (Arranjo de Figuras), visuo-construção e raciocínio espacial (Cubos), síntese e organização perceptiva de estímulos (Armar Objetos; Mader et al., 2004; Simões, 2002). Já em relação às habilidades que requerem velocidade de processamento (Código e Procurar Símbolos) tais como precisão, coordenação visuo-motora, 
aprendizagem automatizada, atenção visual e memória de trabalho visuo-espacial (Mader et al., 2004; Simões, 2002), as diferenças são observadas apenas nas faixas etárias iniciais de $6 \mathrm{e}$ 7 anos, indicando que a idade e o tempo de escolarização podem diminuir as diferenças entre tipos de escola.

Por outro lado, nos subtestes verbais, as diferenças entre os grupos permaneceram ao longo das faixas etárias nos subtestes verbais que requerem conhecimentos gerais, vocabulário, compreensão de situações conhecidas previamente, memória de trabalho auditiva-verbal tanto para manipulação de dígitos quanto para problemas de aritmética e associação semântica (Mader et al., 2004; Simões, 2002). Os subtestes Informação, Semelhanças, Vocabulário e Compreensão demandam habilidades linguísticas e avaliam o nível de conhecimento adquirido no contexto escolar (Simões, 2002). Neste sentido, estes subtestes estão associados à inteligência cristalizada que reflete o tipo de raciocínio desenvolvido a partir das experiências culturais e educacionais (Schelini, 2006).

Essas diferenças encontradas na comparação dos grupos nas diferentes variáveis (subtestes e índices fatoriais) advindas da avaliação de inteligência por meio da WISC-III, mostram que o tipo de escola pode ser um fator importante no desenvolvimento cognitivo das crianças brasileiras devendo ser essa uma variável a ser controlada em estudos futuros e considerada em estudos normativos, assim como realizado por estudos prévios (Almeida \& Primi, 2000; Alves \& Duarte, 2001; Angelini et al., 1999; Bandeira et al., 2004).

Embora a idade seja o principal fator de maturação cerebral, sabe-se que os anos de escolaridade são fortemente correlacionados com a idade e ambos impactam diretamente no desenvolvimento cognitivo e consequentemente na inteligência (Primi et al., 2012). Assim, diante dos resultados obtidos nesse trabalho e observando que, de acordo com a PeNSE 2012 (IBGE, 2012) $82,8 \%$ da população em idade escolar frequenta a escola pública, é necessário que mudanças no sistema de ensino sejam implantadas, a fim de beneficiar o desenvolvimento cognitivo das crianças, uma vez que a inteligência está associada às habilidades demandadas pelo contexto escolar (Gomes, 2010; Pereira, \& Almeida, 2010).

O presente estudo aponta para uma lacuna importante que é a falta de dados normativos distintos para indivíduos pertencentes a escolas públicas e particulares, uma que não há essa distinção na WISC-III (Figueiredo, 2002), bem como na $4^{\mathrm{a}}$ edição, recentemente publicada e comercializada para a população brasileira (Rueda et al., 2013).

\section{Referências}

Almeida, L. S., \& Primi, R. (2000). Bateria de Provas de Raciocínio - BPR-5. São Paulo, SP: Casa do Psicólogo.

Alves, I., \& Duarte, J. (2001). Escala de Maturidade Mental Columbia - Padronização brasileira. São Paulo, SP: Casa do Psicólogo.

Angelini, A. L., Alves, I. C. B., Custódio, E. M., Duarte, W. F., \& Duarte, J. L. (1999). Matrizes Progressivas Coloridas de Raven: Escala Especial. São Paulo, SP: Centro Editor de Testes e Pesquisa em Psicologia.

Bandeira, D. R., Alves, I. C. B., Giacomel, A. E., \& Lorenzatto, L. (2004). Matrizes Progressivas Coloridas de Raven - Escala Especial: Normas para Porto Alegre. Psicologia em Estudo, 9(3), 479486. doi:10.1590/S1413-73722004000300016

Carneiro, P., Meghir, C., \& Parey, M. (2013). Maternal education, home environments and the development of children and adolescents. Journal of the European Economic Association, 11(Suppl. s1), 123-160. doi:10.1111/j.15424774.2012.01096.x

Carroll, J. B. (2012). The Tree-Stratum Theory of Cognitive Abilities. In D. P. Flanagan \& P. L. Harrison (Eds.), Contemporary intellectual assessment: Theories, tests and issues ( $3^{\text {rd }}$ ed., pp. 883-891). New York: The Guilford Press.

Figueiredo, V. L. M. (2002). Escala de Inteligência Wechsler para Crianças e Adolescentes (3. ed.). São Paulo, SP: Casa do Psicólogo.

Flanagan, D. P., \& Kaufman, A. S. (2004). Essentials of WISC-IV Assessment. Hoboken, NJ: John Wiley \& Sons. 
Flores-Mendoza, C. E., \& Nascimento, E. (2007). Condição cognitiva de crianças de zona rural. Estudos de Psicologia (Campinas), 24(1), 1322. doi:10.1590/S0103-166X2007000100002

Gomes, C. M. A. (2010). Avaliando a avaliação escolar: Notas escolares e inteligência fluida. Psicologia em Estudo, 15(4), 841-849. doi:10.1590/ S1413-73722010000400020

Instituto Brasileiro de Geografia e Estatística. (2012). Pesquisa Nacional de Saúde do Escolar. Rio de Janeiro, RJ: Autor.

Klauer, K. J., \& Phye, G. D. (1995). Cognitive training for children: A developmental program of inductive reasoning and problem solving. Toronto, Canada: Hogrefe \& Huber.

Loehlin, J. C. (2000). Group differences in Intelligence. In R. J. Sternberg (Ed.), Handbook of intelligence (pp. 176-193). New York: Cambrigde University Press.

Mader, M. J., Thais, M. E. R. O., \& Ferreira, M. G. R. (2004). Inteligência: Um conceito amplo. In V. M. Andrade, F. H. Santos, \& O. F. A. Bueno (Eds.), Neuropsicologia hoje (pp. 61-76). São Paulo, SP: Artes Médicas.

McGrew, K. S. (2009). CHC Theory and the Human Cognitive Abilities Project: Standing on the shoulders of the giants of psychometric intelligence research. Intelligence, 37(1), 1-10. doi:10.1016/j.intell.2008.08.004

Mecca, T. P. (2010). Tradução, adaptação, fidedignidade e evidências de validade da Bateria de Visualização e Raciocínio da Leiter International Performance Scale Revised (Dissertação de mestrado em Distúrbios do Desenvolvimento, Universidade Presbiteriana Mackenzie, São Paulo, SP, Brasil).

Moura, O., Simões, M. R., \& Pereira, M. (2014). WISC-III cognitive profiles in children with developmental dyslexia: Specific cognitive disability and diagnostic utility. Dyslexia, 20(1), 19-37. doi:10.1002/dys. 1468

Neisser, U. (1998). The rising curve. Washington, DC: American Psychological Association.

Nisbett, R. E., Aronson, J., Blair, C., Dickens, W., Flynn, J., Halpern, D. F., \& Turkheimer, E. (2012). Intelligence: New findings and theoretical developments. American Psychologist, 67(2), 130-159. doi:10.1037/a0026699
Pereira, M., \& Almeida, L. (2010). Predição do rendimento académico no final do ensino secundário na base dos testes de QI na infância. Revista Galego-Portuguesa de Psicoloxía e Educación, 18(1), 239-249.

Primi, R., Couto, G., Almeida, L. S., Guisande, M. A., \& Miguel, F. K. (2012). Intelligence, age and schooling: Data from the Battery of Reasoning Tests (BRT-5). Psicologia: Reflexão e Crítica, 25(1), 79-88. doi:10.1590/S010279722012000100010

Rueda, F. J. M., Noronha, A. P. P., Sisto, F. F., Santos, A. A. A., \& Castro, N. R. (2013). Escala Wechsler de Inteligência para Crianças - WISC-IV. São Paulo, SP: Casa do Psicólogo.

Schelini, P. W. (2006). Teoria das inteligências fluida e cristalizada: Início e evolução. Estudos de Psicologia (Natal), 11(3), 323-332. doi:10.1590/ S1413-294X2006000300010

Schneider, W. J., \& McGrew, K. S. (2012). The Cattell-Horn-Carroll Model of Intelligence. In D. P. Flanagan \& P. L. Harrison (Eds.), Contemporary intellectual assessment: Theories, tests and issues ( $3^{\text {rd }}$ ed., pp. 553-581). New York: The Guilford Press.

Simões, M. R. (2002). Utilizações da WISC-III na avaliação neuropsicológica de crianças e adolescentes. Paidéia (Ribeirão Preto), 12(23), 113132. doi:10.1590/S0103-863X2002000200009

Vista, A. D., \& Grantham, T. C. (2010). Effects of parental education level on fluid intelligence of Philippine public school. Journal of Psychoeducational Assessment, 28(3), 236-248. doi:10.1177/0734282909344416
Recebido: 05/03/2014

$1^{a}$ revisão: $1 \% / 10 / 2014$ Aceite final: 02/10/2014 